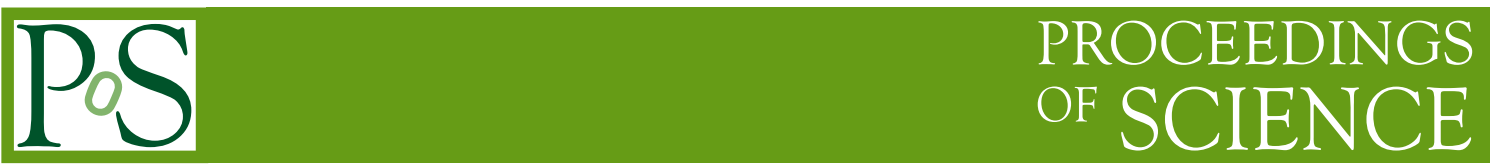

\title{
Overview of Gluon Helicity Measurements at STAR
}

\section{Christopher Dilks*}

for the STAR Collaboration

Pennsylvania State University

E-mail: c jd5150@psu.edu

The contribution to the spin of the proton from the gluon helicity is starting to come into focus: for gluons carrying a large fraction $x$ of the proton momentum, evidence of positive gluon polarization has been observed, via measurements of the longitudinal double-spin asymmetry $A_{L L}$ for inclusive jet and dijet production. $A_{L L}$ is sensitive to the polarized gluon distribution function, $\Delta g(x)$, and while it is positive at high $x$, it is not well constrained for $x<0.05$. Recent measurements at STAR of observables originating dominantly from quark-gluon and gluon-gluon subprocesses aim to improve the precision of $\Delta g(x)$ at high $x$, as well as for the first time provide insight into the low- $x$ contribution. $A_{L L}$ measurements of inclusive jets and dijets at midrapidity $(|\eta|<1)$ and intermediate rapidity $(0.8<\eta<2)$ at STAR at $\sqrt{s}=200$ and $510 \mathrm{GeV}$ will be shown, along with the statuses of ongoing analyses; these measurements will help improve the $\Delta g(x)$ precision for $x \gtrsim 0.01$. Recent $\pi^{0} A_{L L}$ measurements in the forward region $(2.65<\eta<3.9)$ at $\sqrt{s}=510$ $\mathrm{GeV}$ will also be presented, which probe $\Delta g(x)$ down to $x \sim 10^{-3}$. Comparisons of these results to recent global analyses and extrapolations will be discussed.

23rd International Spin Physics Symposium - SPIN2018 -

10-14 September, 2018

Ferrara, Italy

\footnotetext{
${ }^{*}$ Speaker.
} 


\section{Introduction}

The composition of the spin of the proton from the spin and orbital angular momenta of its quarks and gluons is starting to come into focus. By the Jaffe-Manohar spin sum rule [1], the spin $S_{p}$ of the proton is expressed as

$$
S_{p}=\frac{1}{2}=\frac{1}{2} \Delta \Sigma+\Delta G+L,
$$

where $\Delta \Sigma$ is the fractional contribution from the quark spin, $\Delta G$ is that from the gluon helicity, and $L$ is that from the partonic orbital angular momentum. The contributions $\Delta \Sigma$ and $\Delta G$ are integrals over $x$ of the respective helicity distributions, where $x$ is the fraction of the proton momentum carried by the parton. The gluon helicity distribution $\Delta g(x)$ is the difference between the gluon parton distribution function (PDF) for the case of the gluon and proton helicities aligned, minus that of the helicities anti-aligned, and the (anti)quark helicity distributions are also similarly defined.

The quark contribution is constrained to $\Delta \Sigma \sim 0.24[2,3,4,5,6]$. For gluons carrying a large fraction of the proton momentum, $x>0.05$, the gluon helicity contribution is $\Delta G=0.23 \pm 0.06$ [7] and $\Delta G=0.20_{-0.07}^{+0.06}$ [8]; however, $\Delta G$ from gluons with $x<0.05$ is still highly unconstrained and the full gluon helicity integrated over all $x$ is not yet well understood [7, 8]. Moreover, since gluons are vastly dominant in the $x<0.05$ region, it is important to understand their possible contribution to the proton spin. Finally, the partonic orbital angular momentum contribution $L$ has not yet been measured.

The gluon helicity is accessible via measurements of the longitudinal double-spin asymmetry $A_{L L}$, defined as

$$
A_{L L}=\frac{\sigma_{++}-\sigma_{+-}}{\sigma_{++}+\sigma_{+-}}
$$

where $\sigma_{++}\left(\sigma_{+-}\right)$denotes a production cross section, given the colliding proton helicities were the same (opposite). At STAR, $A_{L L}$ is measured for inclusive jet, dijet, and pion production. Recent STAR measurements of $A_{L L}$ in longitudinally polarized proton-proton scattering aim not only to tighten constraints on the magnitude and shape of $\Delta g(x)$ for $x>0.05$, but also aim to probe down to the largely unconstrained low- $x$ region, down to $x \sim 10^{-3}[9,10,11]$.

The proton-proton scattering cross section is collinearly factorizable (see, e.g., [12]), with the initial state protons having contributions from the PDFs $f(x)$ for each parton type $f$ [13]; if the protons are longitudinally polarized, helicity distributions $\Delta f(x)$ contribute to the spin-dependent cross sections $[3,7,8]$. The hard parton scattering process $f_{1} f_{2} \rightarrow f_{3} f_{4}$ is modelled by the hardscattering cross section $\hat{\sigma}\left(k_{i}\right)$ and the parton-level longitudinal double-spin asymmetry $\hat{a}_{L L}\left(k_{i}\right)$, which are both calculable in perturbative QCD and are dependent on the parton momenta $k_{i}[14$, 15]. Finally, for the production of a hadron $h$ from a scattered parton $f_{3}$, there is an additional contribution from the fragmentation function $D_{f_{3}}^{h}(z)$, where $z$ is the fraction of the momentum of $f_{3}$ carried by $h[16]$.

In terms of these distributions, $A_{L L}$ for $h$ production is expressed as

$$
A_{L L}=\frac{\sum \Delta f_{1} \otimes \Delta f_{2} \otimes\left(\hat{\sigma}^{f_{1} f_{2} \rightarrow f_{3} X} \hat{a}_{L L}^{f_{1} f_{2} \rightarrow f_{3} X}\right) \otimes D_{f_{3}}^{h}}{\sum f_{1} \otimes f_{2} \otimes \hat{\sigma}^{f_{1} f_{2} \rightarrow f_{3} X} \otimes D_{f_{3}}^{h}} .
$$


The $\otimes$ symbol denotes convolution and the summations run over the partons $f_{1}$ and $f_{2}$ from the initial protons, as well as the scattered parton $f_{3}$ that fragments to the observable $h$. For jet measurements, no fragmentation functions are needed. All quantities in the right hand side of Eq. 1.3 are well constrained, except for $\Delta g(x)$, in particular in the $x<0.05$ region. Furthermore, measurements of inclusive jets, dijets, and pions at STAR kinematics are dominantly sensitive to quark-gluon and gluon-gluon scattering subprocesses [17, 18, 19], therefore recent measurements of $A_{L L}$ at STAR are sensitive to the gluon helicity.

This presentation focuses on measurements of $A_{L L}$ at the STAR experiment at RHIC. Recent results from three regions of pseudorapidity $\eta$ are presented: central rapidity with $|\eta|<1$, intermediate rapidity with $0.8<\eta<2$, and forward rapidity with $2.65<\eta<3.9$. The data are from longitudinally polarized proton-proton scattering at center-of-mass energies of $\sqrt{s}=200$ and 510 $\mathrm{GeV}$. These measurements, in particular the forward rapidity measurements, are sensitive to gluon $x$ down to $\sim 10^{-3}$. The next three sections summarize the results for these $\eta$ regions, beginning with the central rapidity region.

\section{Central Rapidity Observables}

Measurements of inclusive jets and dijets at central pseudorapidity, with $|\eta|<1$, are sensitive to $x>0.05$ at $\sqrt{s}=200 \mathrm{GeV}$ and $x>0.02$ at $\sqrt{s}=510 \mathrm{GeV}$. The inclusive jet analysis at $\sqrt{s}=$ $200 \mathrm{GeV}$ from 2009 STAR data [20] yielded a positive $A_{L L}$, systematically above the DSSV08 global analysis fit [3], which demonstrates that $\Delta g(x)$ for $x>0.05$ is positive. Inclusive jet $A_{L L}$ measurements at $\sqrt{s}=510 \mathrm{GeV}$ [21] from 2012 and 2013 STAR data show agreement with the 200 $\mathrm{GeV}$ data, but with a sensitivity to somewhat lower $x$, down to 0.02 . This agreement is illustrated in figure 1 , which shows a comparison of the $2009 \sqrt{s}=200 \mathrm{GeV}$ and $2013 \sqrt{s}=510 \mathrm{GeV}$ inclusive jet $A_{L L}$ measurements.

Inclusive dijet measurements probe a much narrower region of $x$, because the partonic $x$ values are calculable from the dijet kinematics. A measurement of dijet $A_{L L}$ from $\sqrt{s}=200 \mathrm{GeV} 2009$ data [23] and a preliminary measurement from $\sqrt{s}=510 \mathrm{GeV}$ 2012-2013 data each show positive asymmetries. These measurements agree with models that predict $\Delta G \sim 0.2$, for two different dijet topologies: the jets 1 and 2 both produced on the same side of the central transverse plane $\left(\operatorname{sign}\left(\eta_{1}\right)=\operatorname{sign}\left(\eta_{2}\right)\right)$, and each jet produced on opposite sides $\left(\operatorname{sign}\left(\eta_{1}\right) \neq \operatorname{sign}\left(\eta_{2}\right)\right)$. Figure 2 shows a comparison of the $2009 \sqrt{s}=200 \mathrm{GeV}$ measurement to that from $2013 \sqrt{s}=510 \mathrm{GeV}$ data, for each of these jet topologies.

\section{Intermediate Rapidity Observables}

Moving forward in $\eta$ to the intermediate region, $0.8<\eta<2$, the sensitivity to gluon $x$ is pushed down to 0.01 for $\sqrt{s}=200 \mathrm{GeV}$; analyses of observables in $\sqrt{s}=510 \mathrm{GeV}$ collisions will push down to $x \approx 0.004$, but are still underway. The measurement of $A_{L L}$ in inclusive dijets at 200 $\mathrm{GeV}$ [11] from 2009 data provides tighter constraints on the size and especially the shape of $\Delta g(x)$. This is a newly published result and was presented at this conference; for further details, see the presentation and proceedings by T. Lin [24]. 


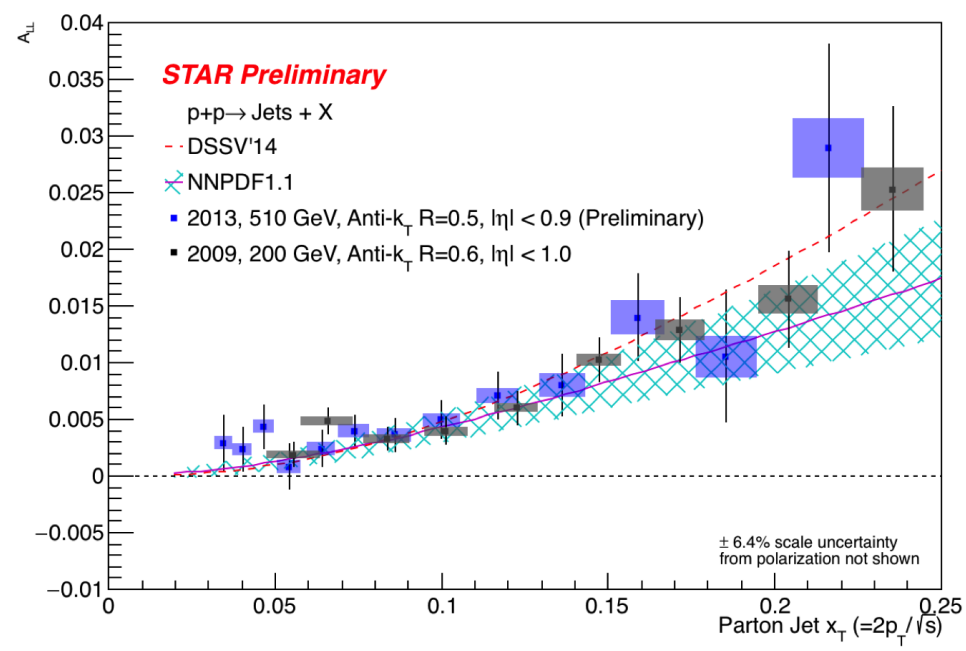

Figure 1: Comparison of central inclusive jet $A_{L L}$ at $\sqrt{s}=200 \mathrm{GeV}$ from 2009 STAR data (black points) [20] to a preliminary measurement at $\sqrt{s}=510 \mathrm{GeV}$ from 2013 (blue points) [21]. The vertical lines represent statistical uncertainties and the shaded boxes represent systematic uncertainties. The DSSV14 [8] and NNPDFpol1.1 [7] global fit curves are shown as well. From [22].
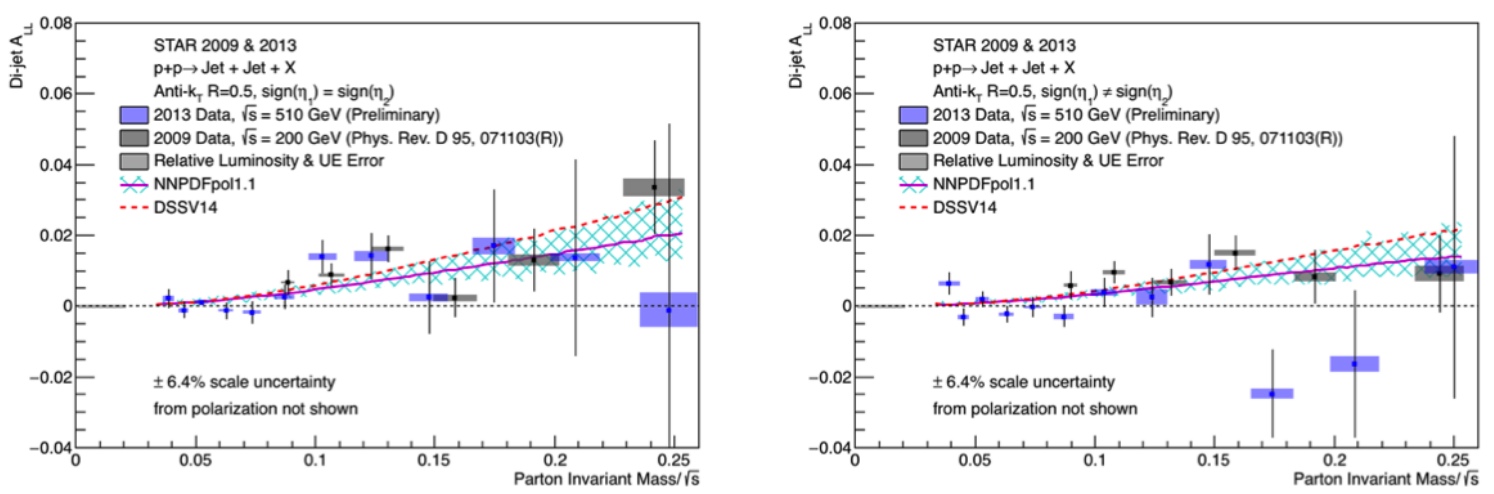

Figure 2: Comparison of central inclusive dijet $A_{L L}$ at $\sqrt{s}=200 \mathrm{GeV}$ from 2009 STAR data (black points) [23] to a preliminary measurement at $\sqrt{s}=510 \mathrm{GeV}$ from 2013 (blue points). The two panels are for the different dijet topologies: the left is for $\operatorname{sign}\left(\eta_{1}\right)=\operatorname{sign}\left(\eta_{2}\right)$ and the right is for $\operatorname{sign}\left(\eta_{1}\right) \neq \operatorname{sign}\left(\eta_{2}\right)$. The vertical lines represent statistical uncertainties and the shaded boxes represent systematic uncertainties. The DSSV14 [8] and NNPDFpol1.1 [7] global fit curves are shown as well. From [22].

Three dijet topologies were assessed: (1) one jet at intermediate rapidity and the second jet at central rapidity, on the opposite side of the central plane as the first jet, (2) one jet at intermediate rapidity and the second central jet on the same side of the central plane, and (3) both jets at intermediate rapidity. Case (3) probes the lowest $x$ of the three topologies and has a large $A_{L L}$ systematically above the DSSV14 [8] and NNPDFpol1.1 [7] theoretical predictions, whereas cases (1) and (2) show rather good agreement with theory. The analysis of $510 \mathrm{GeV}$ dijets from 2012 and 2013 data, which will probe down to $x \approx 0.004$, is currently underway.

A somewhat older, but related result is the intermediate rapidity $\pi^{0} A_{L L}$ measurement from 


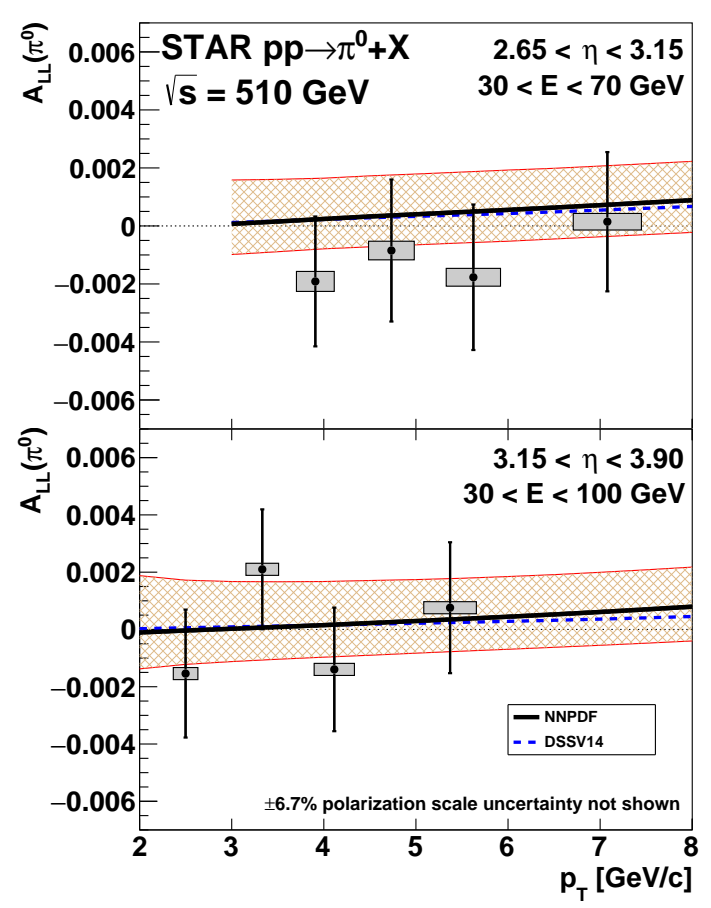

Figure 3: Forward $\pi^{0} A_{L L}$ for inner $\eta$ region (top) and outer $\eta$ region (bottom). See text for details. From [10].

$\sqrt{s}=200 \mathrm{GeV}$ data from 2006 [25]. This result agrees with the available models, but is not significantly capable of distinguishing between them. Analysis of $\sqrt{s}=510 \mathrm{GeV} \pi^{0}$ data is also underway.

\section{Forward Rapidity Observables}

Forward observables are sensitive to gluons of even lower $x$, since the dominant subprocess is a soft low- $x$ gluon scattering off a hard mid-to-high- $x$ quark. The forward $\pi^{0} A_{L L}$ in the pseudorapidity range $2.65<\eta<3.9$ probes gluon $x$ down to $10^{-3}$ [10]. This result has also been recently published, and some more details are provided here.

Figure 3 shows the forward $\pi^{0} A_{L L}$ measurement, along with extrapolations of $A_{L L}$ predictions to these forward kinematics, assuming the central $\Delta g(x)$ values from the recent DSSV14 [8] and NNPDFpol1.1 [7] global analyses. The top panel is for $\pi^{0}$ production in the pseudorapidity range $2.65<\eta<3.15$ and the bottom panel is for the range $3.15<\eta<3$.9. The vertical error bars on the data represent the statistical uncertainty, the horizontal extent of the shaded gray boxes represents the systematic uncertainty on $p_{T}$, and the vertical extent represents the systematic uncertainty on $A_{L L}$; an additional $6.7 \%$ scale uncertainty from the polarization is not shown.

The dashed blue curve represents the DSSV14 extrapolation, and the solid black curve along with the hatched uncertainty band represents the NNPDFpol1.1 extrapolation. The uncertainty band was determined by calculating $A_{L L}$ at a given $x$ for each of the 100 replica PDFs, and taking 


\begin{tabular}{|c|c|c|c|c|c|c|c|}
\hline \multirow{2}{*}{$\sqrt{s} \mathbf{G e V}$} & \multirow{2}{*}{ Year } & \multicolumn{2}{|c|}{ Central $\eta$} & \multicolumn{2}{|c|}{ Intermediate $\eta$} & \multicolumn{2}{|c|}{ Forward $\eta$} \\
\hline & & Jets & Dijets & Dijets & Pions & Pions & Dijets \\
\hline \multirow{6}{*}{200} & \multirow{2}{*}{2006} & Published & & & Published & & \multirow{6}{*}{$\mathrm{n} / \mathrm{a}$} \\
\hline & & $x>0.05$ & & & $x>0.01$ & & \\
\hline & \multirow{2}{*}{2009} & Published & Published & Published & & & \\
\hline & & $x>0.05$ & $x>0.05$ & $x>0.01$ & & & \\
\hline & \multirow{2}{*}{2015} & Underway & Underway & & & Underway & \\
\hline & & $x>0.05$ & $x>0.05$ & & & $x>0.0025$ & \\
\hline \multirow{4}{*}{510} & \multirow{2}{*}{2012} & Preliminary & Preliminary & Underway & Underway & Published & \multirow{4}{*}{$\mathrm{n} / \mathrm{a}$} \\
\hline & & $x>0.02$ & $x>0.02$ & $x>0.004$ & $x>0.004$ & $x>0.001$ & \\
\hline & \multirow{2}{*}{2013} & Preliminary & Preliminary & Underway & Underway & Published & \\
\hline & & $x>0.02$ & $x>0.02$ & $x>0.004$ & $x>0.004$ & $x>0.001$ & \\
\hline \multirow{2}{*}{200 and 510} & \multirow{2}{*}{$2021+$} & & & & & & Future \\
\hline & & & & & & & $x>0.001$ \\
\hline
\end{tabular}

Table 1: Summary of STAR $A_{L L}$ analyses, sensitive to gluon helicity, along with $x$ sensitivity for each analysis.

the standard deviation of this distribution. The data points agree with the extrapolated theory curves, within statistical and systematic uncertainties. Nonetheless, they will help constrain the size of $\Delta g(x)$ down to $x \sim 10^{-3}$ in the upcoming global analysis.

Looking ahead to the future, upgrade plans for a forward calorimeter and tracking system at STAR are underway [26]; see also the presentation and proceedings by $\mathrm{K}$. Barish [27]. The capability of measuring forward jets and dijets will also probe $x$ down to $\sim 10^{-3}$, but will more precisely probe the shape of $\Delta g(x)$ in this important region. Forward tracking detectors will be installed inside the STAR Time Projection Chamber for tracking forward charged hadrons. A new electromagnetic calorimeter as well as a new hadronic calorimeter behind it will take the place of the recently decommissioned Forward Meson Spectrometer, and when combined with the new forward tracking system, will open the doors to many more detailed forward measurements.

The next global analysis will likely include many of these newly published results. The forward $\pi^{0}$ s and intermediate dijets will help constrain $\Delta g(x)$ in the important low- $x$ region, where the gluons are vastly dominant in the overall parton densities. The central jets and dijets will help pin down the size and especially shape for $x>0.02$, and altogether a more accurate determination of the gluon helicity contribution to the proton is expected in the near future.

\section{Summary}

Table 1 summarizes the recent STAR $A_{L L}$ measurement statuses and gluon $x$ sensitivities. The $\sqrt{s}=200 \mathrm{GeV}$ results are shown in the top half, followed by the $510 \mathrm{GeV}$ results. The columns are organized by pseudorapidity region, with observables within each region. For each analysis, the progression of analysis status is from "underway," meaning the analysis is being actively worked on but no results have been released by STAR, to "preliminary," meaning a first look at the measurement has been released, but more work needs to be done before finalizing it, and finally to "published," meaning the result has been finalized and published. The last row is for the future spin program in $2021+$ with the new forward upgrade, giving access to forward dijets. 


\section{References}

[1] R. Jaffe and A. Manohar, Nucl. Phys. B 337, 509 (1990).

[2] D. de Florian, R. Sassot, M. Stratmann and W. Vogelsang, Phys. Rev. Lett. 101, 072001 (2008).

[3] D. de Florian, R. Sassot, M. Stratmann and W. Vogelsang, Phys. Rev. D 80, 034030 (2009).

[4] J. Blümlein and H. Böttcher, Nucl. Phys. B 841, 205 (2010).

[5] E. Leader, A. V. Sidorov and D. B. Stamenov, Phys. Rev. D 82, 114018 (2010).

[6] R. D. Ball et al. [NNPDF Collaboration], Nucl. Phys. B 874, 36 (2013).

[7] E. Nocera et al. [NNPDF Collaboration], Nucl. Phys. B 887, 276 (2014).

[8] D. de Florian, R. Sassot, M. Stratmann and W. Vogelsang, Phys. Rev. Lett. 113, 012001 (2014).

[9] E. C. Aschenauer, R. Sassot and M. Stratmann, Phys. Rev. D 92, 094030 (2015).

[10] J. Adam et al. [STAR Collaboration], Phys. Rev. D 98, 032013 (2018).

[11] J. Adam et al. [STAR Collaboration], Phys. Rev. D 98, 032011 (2018).

[12] G. Bunce, N. Saito, J. Soffer and W. Vogelsang, Ann. Rev. Nucl. Part. Sci. 50, 525 (2000).

[13] S. Dulat et al., Phys. Rev. D 93, 033006 (2016).

[14] J. Babcock, E. Monsay and D. W. Sivers, Phys. Rev. D 19, 1483 (1979).

[15] N. S. Craigie, K. Hidaka, M. Jacob and F. M. Renard, Phys. Rept. 99, 69 (1983).

[16] C. W. Kao, S. I. Nam, F. J. Jiang and D. J. Yang, EPJ Web Conf. 66, 06008 (2014).

[17] S. Kretzer, Acta Phys. Polon. B36, 179 (2005).

[18] E.C. Aschenauer et al., The RHIC spin program: achievements and future opportunities, (2015).

[19] C. Aidala, G. Bunce et al., Research plan for spin physics at RHIC, (2005).

[20] L. Adamczyk et al., Phys. Rev. Lett. 115, 092002 (2015).

[21] Z. Chang, Int. J. Mod. Phys. Conf. Ser. 40, 1660021 (2016).

[22] A. Quintero, PoS DIS2018, 144 (2018).

[23] L. Adamczyk et al., Phys. Rev. D 95, 071103 (2017).

[24] T. Lin, in these proceedings (2018).

[25] L. Adamczyk et al. [STAR Collaboration], Phys. Rev. D 89, 012001 (2014).

[26] STAR Collaboration, The star forward calorimeter system and forward tracking system proposal, STAR Note SN0648, (2017).

[27] K. Barish, in these proceedings (2018). 